\title{
Tracheostomy and Gastrostomy in Geriatric Trauma Associated With High Postdischarge Mortality
}

\author{
Sherry M. Boone, $\mathrm{MSN}^{1}$; Bryan R. Collier, DO ${ }^{1,2}$; Emily R. Faulks, MD ${ }^{1,2}$; Tonja M. Locklear, PhD ${ }^{3}$; \\ Katie L. Bower, $\mathrm{MD}^{1,2}$; Daniel I. Lollar, $\mathrm{MD}^{1,2}$; Nitasha Dhiman, $\mathrm{MD}^{2}$; Michael S. Nussbaum, $\mathrm{MD}^{1,2}$; \\ Mark E. Hamill, MD ${ }^{1,2}$
}

Objectives: Identify 5-year mortality rates in trauma patients greater than 18 years old who undergo tracheostomy and/or gastrostomy tube placement.

Design: Retrospective convenience sample with two cohorts.

Setting: Academic level 1 trauma center.

Patients: Hospitalized patients admitted to the trauma service from July 2008 to December 2012 who underwent tracheostomy and/or gastrostomy tube placement.

Interventions: Patients were placed into two cohorts: adult 18-64 and geriatric greater than or equal to 65 ; mortality data were obtained from the National Death Index.

Measurements and Main Results: The primary outcome was 5-year mortality of both cohorts as well as those admitted who did not receive tracheostomy or gastrostomy. Univariate analysis was performed using Fisher exact and Wilcoxon signed-rank tests. Kaplan-Meier curves were plotted to examine mortality up to 5 years after discharge.

Conclusions: Five-year postdischarge mortality is significantly higher in geriatric patients undergoing tracheostomy and/or gastrostomy after traumatic injury. Fifty percent die within the first 28 weeks following discharge and 93\% die within 2 years.

Key Words: gastrostomy; geriatrics; mortality; tracheostomy; trauma

'Department of Surgery, Division of Trauma and Surgical Critical Care, Carilion Clinic, Roanoke, VA

${ }^{2}$ Department of Surgery, Virginia Tech Carilion School of Medicine, Roanoke, VA

${ }^{3}$ Health Analytics, Carilion Clinic, Roanoke, VA.

Current address for Dr. Dhiman: Department of Radiology, Columbia University, New York, NY.

Copyright (c) 2020 The Authors. Published by Wolters Kluwer Health, Inc. on behalf of the Society of Critical Care Medicine. This is an open-access article distributed under the terms of the Creative Commons Attribution-Non Commercial-No Derivatives License 4.0 (CCBY-NC-ND), where it is permissible to download and share the work provided it is properly cited. The work cannot be changed in any way or used commercially without permission from the journal.

Crit Care Exp/ 2020; 2:e0156

DOI: $10.1097 / C C E .0000000000000156$
The geriatric population in America, defined as age 65 old or older, is on the rise. This population grew by 1.6 million from 2014 to 2015 and is projected to increase from 47.8 to 98.2 million by 2060 (1). Given the aging population, trauma centers are seeing a significant increase in older patients with traumatic injuries. In 2013 alone, geriatric patients greater than 65 years old made up $23 \%$ of the total trauma admissions nationwide (2). Identifying how various practices and procedures affect the outcomes of this age group will ensure that we offer the best care following traumatic events. Geriatric patients have been identified as an at-risk population with longer lengths of stay, requiring more resources postdischarge, and with an inpatient mortality rate three times that of the nongeriatric population (3).

The purpose of this study was to assess the long-term mortality of adult and geriatric trauma patients who undergo tracheostomy and/or gastrostomy tube placement after severe trauma. Our hypothesis is that geriatric trauma patients who undergo tracheostomy and/or gastrostomy tube placement will have significantly worse mortality after hospital discharge than adult patients undergoing similar procedures.

\section{MATERIALS AND METHODS}

Following institutional review board approval, the trauma registry of our academic level 1 trauma center was queried for trauma patients 18 years old or older. Patients evaluated between July 2008 and December 2012 were selected and separated into two cohorts: adults (age 18-64) and geriatric (age $\geq 65$ ). Patients who underwent tracheostomy and/or gastrostomy tube placement were identified. Patients with multiple admissions were excluded. Five-year mortality data was then obtained by cross-referencing this patient population with information from the National Death Index. Univariate analysis was performed using Fisher exact and Wilcoxon signed-rank tests. Kaplan-Meier curves were plotted to examine 5-year mortality. This study was approved by the institutional review board at Carilion Clinic with a waiver of informed consent. 


\section{RESULTS}

After excluding those with multiple admissions, 7,916 patients were identified. The sample was comprised of 5,861 (74.0\%) adult and 2,055 (26.0\%) geriatric patients. Patients undergoing tracheostomy and/or gastrostomy tube placement were identified totaling 129 between the two cohorts. Tracheostomy and/or gastrostomy tube placement was performed in 81 adult patients (1.38\%) and 48 geriatric patients (2.34\%). In patient mortality for adults was $6.2 \%(5 / 81)$ following these procedures, which is significantly lower $(p=0.02)$ compared with the geriatric in patient mortality of $20.8 \%$ (10/48).

All patients with tracheostomy and/or gastrostomy had statistically higher $(p<0.0001)$ postdischarge mortality than the group without these procedures (Fig. 1). In up to 5 years after discharge, postdischarge mortality in the geriatric and adult cohort who received a tracheostomy and/or gastrostomy reached $63.2 \%(24 / 38)$ and $10 \%(7 / 69)$, respectively, which was significantly higher $(p<0.0001$ and $p=0.001$, respectively) than the geriatric and adult patients who did not receive those procedures (Figs. 2 and 3). In patients who received a tracheostomy and/or gastrostomy, the relative risk of mortality for geriatric patients postdischarge was found to be 6.9 times higher than adults (95\% CI, 3.25-14.47). In the geriatric population, comparison between those receiving who had tracheostomy and/or gastrostomy tube placement and those who did not reveal a higher Injury Severity Score (ISS), overall length of stay (LOS), ICU stay, need for ICU admission, \% with head injuries, median Abbreviated Injury Scale (AIS) head/neck, \% with chest injuries, median AIS chest, and median AIS external (Table 1). In the geriatric patient cohort, we assessed time from discharge to death. We found $25 \%$ mortality by 23 days ( $95 \%$ CI, 0-59 d) after discharge and $50 \%$ by 196 days (95\% CI, 43-617 d). Only 7\% of the patients who die within 5 years actually live longer than 2 years after discharge. For the geriatric patients without tubes, we found $25 \%$ mortality by 285 days ( $95 \%$ CI, 203-376 d). Further survival analysis was performed on the geriatric cohort to determine if the postdischarge mortality effect was related to severity of injury alone. When examined based on lower injury burden (ISS $\leq 15$ ) or severe injury burden (ISS $\geq 16$ ), a significantly higher postdischarge mortality was observed with those receiving tracheostomy and/or gastrostomy tube placement in both groups ( $p$ values of 0.0022 and 0.0018 , respectively).

Overall mortality, including both inpatient and postdischarge, was significantly higher in the geriatric group $(70.8 \%, 34 / 48)$ 5 -year mortality versus the adult cohort $(14 \%, 12 / 81)$. The geriatric patients who died in the 5 years following tracheostomy and/ or gastrostomy were older with a median age of 78 versus 71.5 and had lower AIS chest of 0 versus 3; however, had similar ISS, ventilator days, and ICU and hospital LOS when compared with survivors in the geriatric cohort (Table 2).

In addition to the effect of age on mortality, a trend was observed based on the specific procedures performed (Fig. 4). The cohort of all patients that received gastrostomy tubes were found to have higher mortality $(61.5 \%, 16 / 26 ; p=0.004)$ than the cohorts who received a tracheostomy tube alone $(15.7 \%, 11 / 70)$ or a tracheostomy and gastrostomy tubes $(22.2 \%, 4 / 18)$.

\section{DISCUSSION}

In this investigation, we found that geriatric patients admitted following severe trauma that undergo tracheostomy and/or gastrostomy have significantly higher 5-year mortality when compared with nongeriatric adults undergoing similar procedures. Although this is significant both for the inpatient and postdischarge periods, perhaps the most concerning data discovered is the timeframe in which the geriatric patients died after discharge. Of the 24 geriatric patients that survived to discharge, $25 \%$ died in less than 1 month and 50\% died in less than 7 months. Only $7 \%$ of those discharged, who died within 5 years remained alive longer than 2 years. In addition, we demonstrated a trend in mortality based on the specific procedure performed. Patients undergoing gastrostomy tube placement only demonstrated considerably higher mortality than those undergoing
Figure 1. Kaplan-Meier curves displaying the survival probability for adult and geriatric patients who underwent tracheostomy and/or gastrostomy tube placement after suffering traumatic injuries. Postdischarge mortality in the geriatric cohort was $63.2 \%(24 / 38)$ versus adults $(10 \%, 7 / 69)$. 


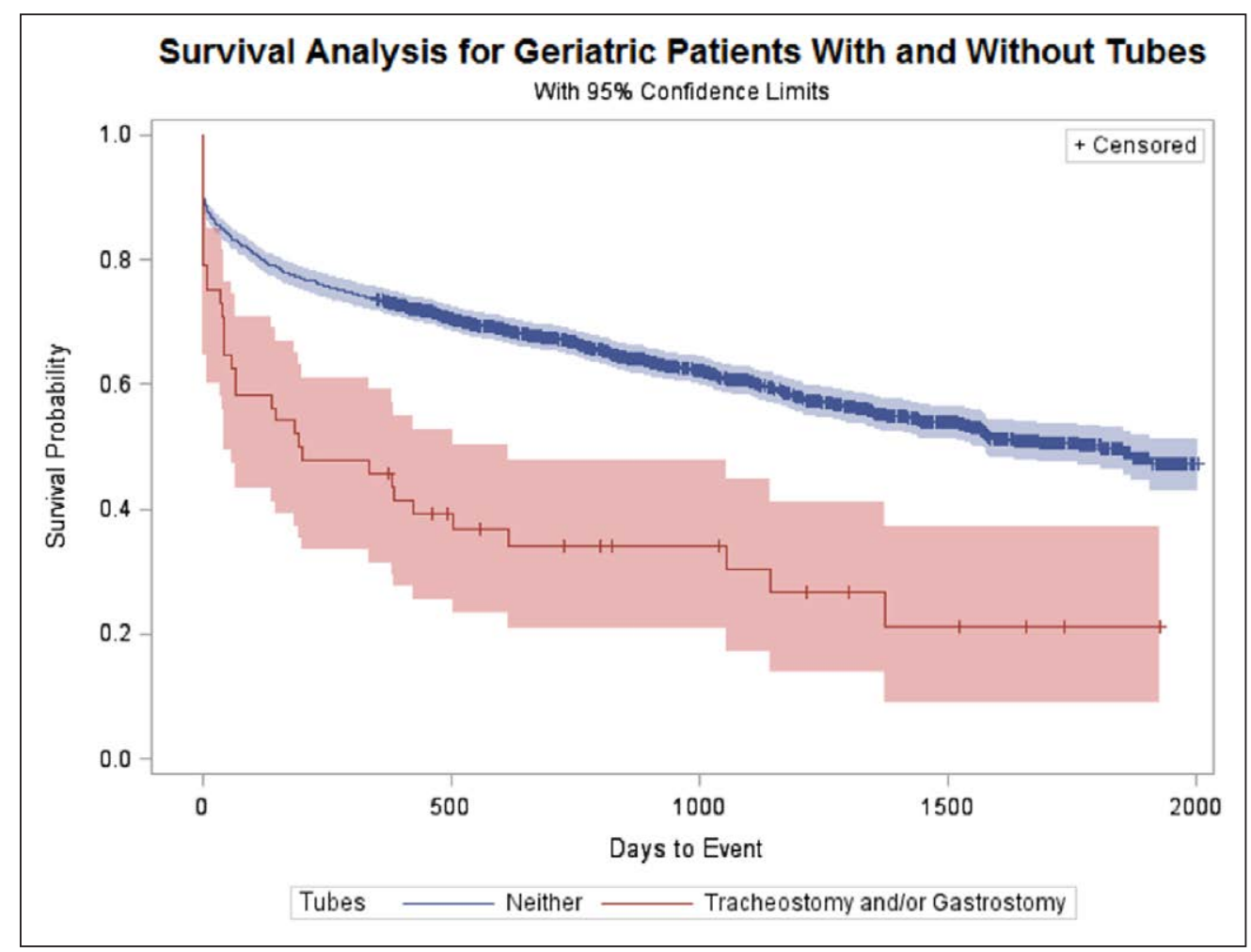

Figure 2. Kaplan-Meier curve displaying postdischarge survival probability of geriatric patients' post-traumatic event undergoing tracheostomy and/or gastrostomy tube placement versus those not undergoing procedures. Twenty-four of the 38 geriatric patients with tubes died within $5 \mathrm{yr}$ of discharge.

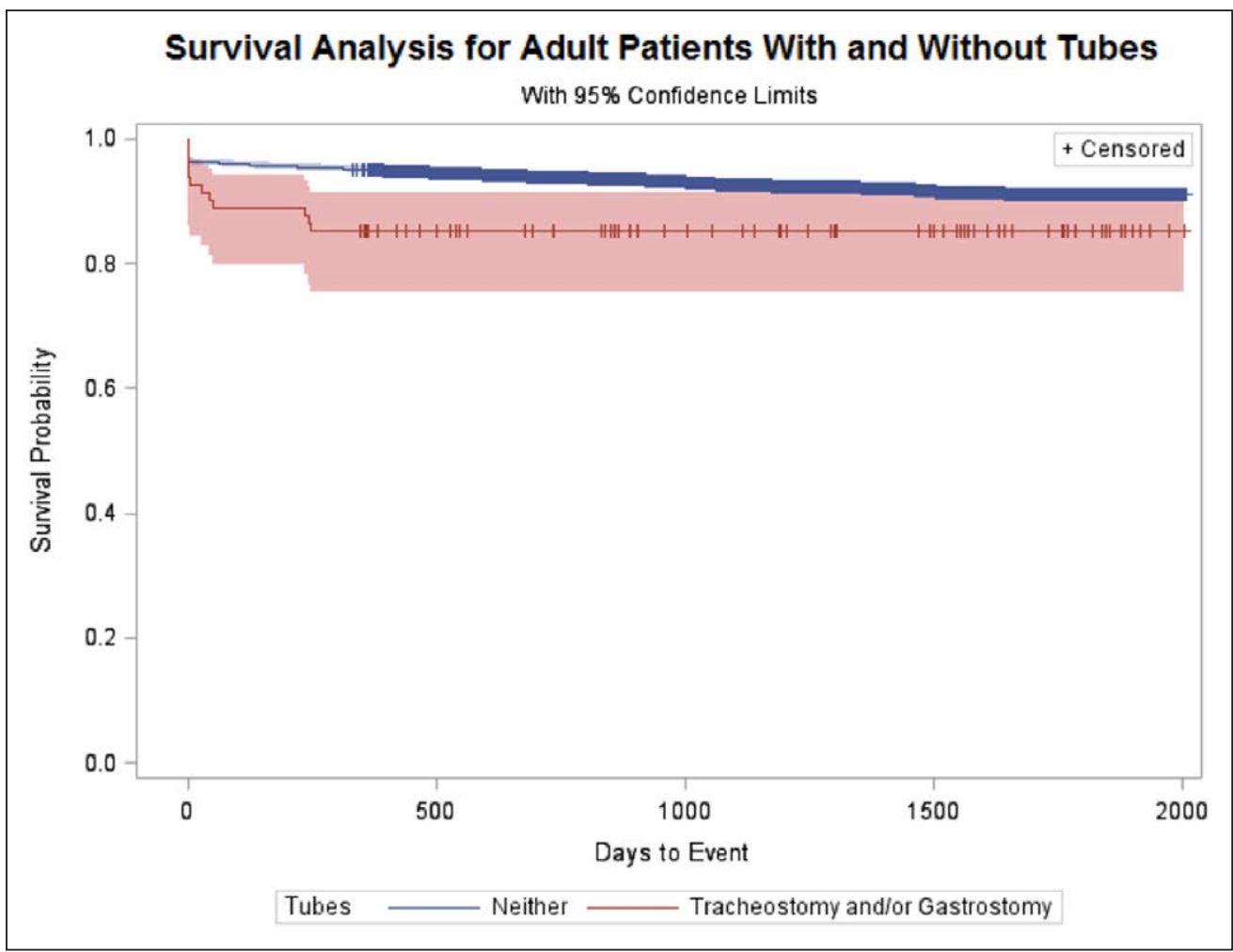

Figure 3. Kaplan-Meier curve displaying postdischarge survival probability of adult patients post-traumatic event undergoing tracheostomy and/or gastrostomy tube placement versus those not undergoing procedures. Seven out of the 76 adult patients with tubes died within the $5 \mathrm{yr}$ postdischarge. tracheostomy either alone or in combination with gastrostomy, irrespective of the patient's age. This is a concerning trend that requires further evaluation over a larger sample. Evaluating causes of death would assist in identifying risk factors that may lead to higher mortality. One interesting finding is that geriatric patients who survived after tracheostomy and/or gastrostomy tube placement had a significantly higher AIS chest than those who died in the postdischarge period. Although it is difficult to ascertain the exact cause from our data, it likely represents a survivor bias where those with more severe chest injuries died during their index admission and did not survive to the postdischarge period.

Tracheostomy and gastrostomy tube placement are common procedures following severe trauma. Tracheostomy benefits include improved oral hygiene and secretion removal and may also improve communication and patient comfort (4). Early versus late tracheostomy placement has been heavily studied to assess benefits based on timing. The definition of early tracheostomy ranges from 2 to 10 days after intubation (5). Some studies have found that early tracheostomy placement can offer benefits including decreased ventilator days, rates of ventilator-associated pneumonia, and ICU and overall hospital LOS; however, it has not been associated with a decrease in mortality (6-9). Barquist et al (4) performed a randomized prospective study finding that ventilator days, pneumonia rates, and ICU LOS were not decreased by the tracheostomy being performed by day 8 . The definitive answer on timing of tracheostomy placement remains unanswered.

Adequate nutritional support is imperative for healing following severe trauma. Although many trauma patients are well-nourished prior to their event, leading up to hospitalization, this is not always the case. Some patients may present with protein-calorie malnutrition; they 
TABLE 1. Postdischarge 5-Year Survival Comparison for Geriatric Patients With and Without Tracheostomy and/or Gastrostomy

\begin{tabular}{|c|c|c|c|}
\hline Group Characteristic & $\begin{array}{c}\text { Tracheostomy } \\
\text { and/or } \\
\text { Gastrostomy } \\
\text { Tube }\end{array}$ & $\begin{array}{l}\text { No } \\
\text { Tubes }\end{array}$ & $p$ \\
\hline$N$ & 38 & 1,808 & \\
\hline Median age & 77 & 78 & 0.6605 \\
\hline Median Injury Severity Score & 17 & 10 & $<0.0001^{\mathrm{a}}$ \\
\hline $\begin{array}{l}\text { Median hospital length } \\
\text { of stay }\end{array}$ & 18 & 4 & $<0.0001^{\mathrm{a}}$ \\
\hline Median ICU stay & 9.5 & 3 & \\
\hline$\%$ in ICU & 94.74 & 28.65 & $<0.0001^{a}$ \\
\hline Median ventilator days & 9 & 5 & \\
\hline$\%$ on ventilator & 60.53 & 8.63 & 0.0964 \\
\hline Median AIS head/neck & 4 & 3 & \\
\hline$\%$ with head/neck injury & 76.32 & 52.60 & $0.0476^{a}$ \\
\hline Median AIS face & 2 & 2 & \\
\hline$\%$ with face injury & 26.32 & 14.38 & 0.1424 \\
\hline Median AIS chest & 3 & 3 & \\
\hline$\%$ with chest injury & 36.84 & 32.47 & $0.0018^{a}$ \\
\hline Median AIS abdomen/pelvis & 2 & 2 & \\
\hline $\begin{array}{l}\% \text { with abdomen/ } \\
\text { pelvis injury }\end{array}$ & 13.16 & 15.04 & 0.5374 \\
\hline Median AIS extremity & 2 & 2 & \\
\hline$\%$ with extremity injury & 31.58 & 30.25 & 0.1587 \\
\hline Median AIS external & 1 & 1 & \\
\hline$\%$ with external injury & 34.21 & 50.17 & $0.0424^{a}$ \\
\hline Caucasian \% & 92.11 & 94.63 & 0.4574 \\
\hline Male \% & 57.89 & 46.24 & 0.1885 \\
\hline Blunt \% & 94.74 & 97.51 & 0.2519 \\
\hline
\end{tabular}

AIS $=$ Abbreviated Injury Scale.

${ }^{a} p<0.05$.

include patients with multiple comorbidities, those with substance abuse, and geriatric patients (10). Early enteral nutrition support following severe illness may decrease complications and length of ICU stay and is recommended in the current guidelines published by the American Society for Parenteral and Enteral Nutrition. The current recommendations include initiating enteral nutrition support within 24-48 hours in critically ill patients when they are unable to maintain their nutritional needs orally (11). Patients who are unable to consume nutrition by mouth have many options for supplemental support via orogastric, nasogastric, nasoduodenal, or nasojejunal tubes which may be placed. Nasoenteric or oral-enteric tubes are designed for short-term support and formal gastrostomy
TABLE 2. Postdischarge 5-Year Survival Comparison for the 38 Geriatric Patients with Tracheostomy and/or Gastrostomy

\begin{tabular}{lccc} 
Group Characteristic & Alive & Dead & $\boldsymbol{p}$ \\
\hline$N$ & 14 & 24 & \\
Median age & 71.5 & 78 & $0.0170^{\mathrm{a}}$ \\
Median Injury Severity Score & 18.5 & 16.5 & 0.8917 \\
Median hospital length of stay & 18.5 & 18 & 0.3454 \\
Median ICU stay & 9.5 & 8 & 0.7068 \\
Median ventilator days & 3.5 & 1 & 0.8402 \\
Median AIS head/neck & 2.5 & 4 & 0.2003 \\
Median AIS face & 0 & 0 & 0.3375 \\
Median AIS chest & 3 & 0 & $0.0326^{a}$ \\
Median AIS abdomen/pelvis & 0 & 0 & 0.4905 \\
Median AIS extremity & 0 & 0 & 0.6713 \\
Median AIS external & 0 & 0 & 0.5498 \\
Caucasian \% & 100.00 & 87.50 & 0.2831 \\
Male \% & 71.43 & 50.00 & 0.3087 \\
Blunt \% & 92.86 & 100.00 & 0.3684 \\
\hline
\end{tabular}

AIS $=$ Abbreviated Injury Scale.

${ }^{\mathrm{a} p} p<0.05$.

should be considered when nutritional support is likely to be needed greater than 4 weeks (12).

It is known that tracheostomy and gastrostomy tube placement after severe trauma may assist in facilitating hospital discharge. A shorter LOS will likely decrease overall hospitalization costs. However, the financial burden for these patients and their families postdischarge should be considered when comparing benefits of early procedures. The U.S. Department of Health published the cost of a skilled nursing facility in 2016 to be on average $\$ 6,844$ / month (13). Fifty percent of those patients who die within five years do so in only seven months. That equates to almost $\$ 48,000$ being spent for care in the last seven months of life, not including the initial hospitalization.

In 2017, the American College of Surgeons (ACS) Trauma Quality Improvement Program recognized the importance of palliative care in trauma, developing best practice guidelines. Given the short duration of life expectancy in postoperative tracheostomy and gastrostomy geriatric patients, palliative care may be beneficial in both symptom management and decision-making prior to the procedure. These best practice guidelines suggest providing palliative care along with life-sustaining trauma care throughout the recovery process. The team providing optimal palliative care requires an interdisciplinary approach. A major focus of the team should be on importance of communication (14). These best practice guidelines should be implemented in the discussions held with the patient and/or family members when discussing long-term support measures including tracheostomy and gastrostomy placement regardless of the patients' age. 


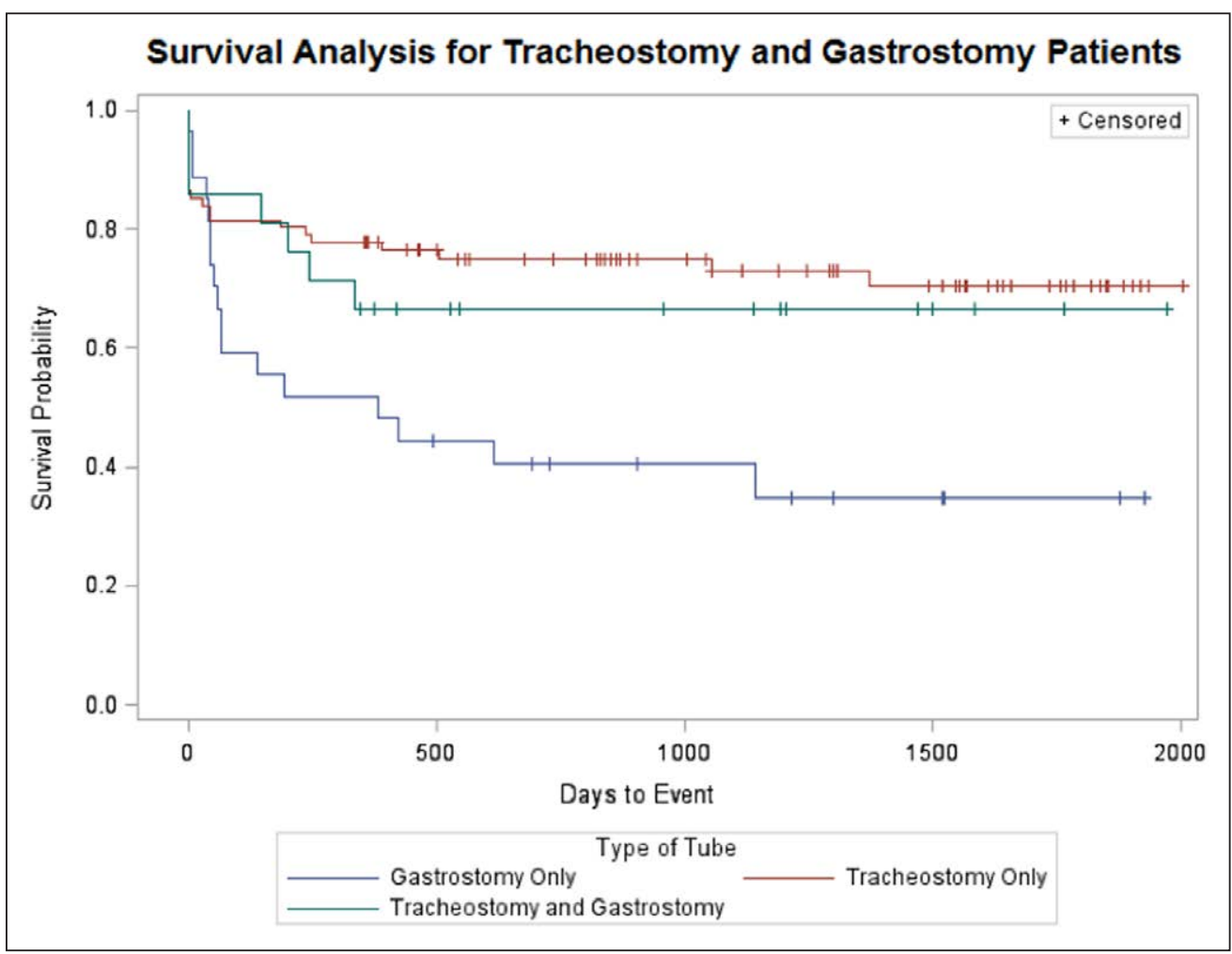

Figure 4. Kaplan-Meier curves for cumulative survival probability for all patients undergoing tracheostomy, gastrostomy, and versus tracheostomy and gastrostomy procedures. Mortality was highest in the gastrostomy cohort at $61.5 \%(16 / 26)$ when compared to tracheostomy $15.7 \%(11 / 70)$ or tracheostomy and gastrostomy $22.2 \%(4 / 18)$ cohorts.

The ACS also published guidelines regarding geriatric trauma patients. They state that more than $40 \%$ of patients must make decisions regarding care near the end of life. They estimate nearly $70 \%$ lack the capacity to make decisions during this time, requiring surrogate decision-makers to step in. Identifying surrogate decisionmakers, including power of attorney and next of kin, is an important step in one's care following severe trauma. If a patient has capacity at admission, goals of care should be discussed, including desires for life prolonging measures and surrogate decision-makers, and confirmed with the patient. Frailty scores may be beneficial in predicting return of previous functional status following a traumatic event (15).

Our study does have several important limitations. To start, a limitation of this retrospective study was the inability to evaluate cause of death from the National Death Index. No data was available regarding the patient's quality of life or functional status during the time preceding death. We also lacked data on the patient's location prior to death, for example, home versus facility. In addition, as a retrospective study, we are able to demonstrate only associationand the specific causes of death cannot be fully established. Finally, due to our small sample size, the ability to generalize our study is limited. However, given our compelling results, our hope is that this will severe to generate further research on the topic.

\section{CONCLUSIONS}

With the elderly population on the rise in the United States, trauma centers can expect to treat more geriatric trauma patients in the coming years. Providing excellent care to this patient population should

\section{ACKNOWLEDGMENTS} Medicine, Roanoke, Virginia. ruary $25-28,2018$. interest.

\section{REFERENCES} 137-150 include a multidisciplinary approach, including specialized nurses, therapists, gerontologists, and palliative care providers. Severely injured patients may benefit from life prolonging procedures including tracheostomy and gastrostomy tube placement. Further research is needed to assess the risks and benefits of these procedures in the geriatric population. Identifying commonalities in the geriatric patients who survive 5 years postdischarge and disease or injury patterns in those who die within months of discharge may assist in improving patient selection for these procedures. Given the high mortality of geriatric patients in the months immediately following discharge, it is important to consider quality of life in the decision-making process regarding tracheostomy and gastrostomy tube placement. Additional research is needed to help identify the subset of patients who would have the most long-term benefits and to guide healthcare professionals in providing the best possible care to the at-risk population.

The authors would like to acknowledge the Virginia Tech Open Access Subvention Fund for assistance with payment of the article processing charges after the manuscript was accepted.

This work was performed at Carilion Clinic, Virginia Tech Carilion School of

Presented, in part, at the 47th Critical Care Congress, San Antonio, TX, Feb-

The authors have disclosed that they do not have any potential conflicts of

For information regarding this article, E-mail: hamillm@mac.com

1. Huntley-Hall N; United States Census Bureau: Profile America Facts for Features: CB17-FF.08. Nadine Huntley-Hall. 2017. Available at: https:// census.gov/content/dam/Census/newsroom/facts-for-features/2017/ cb17-ff08.pdf. Accessed February 18, 2018

2. Bonne S, Schuerer DJ: Trauma in the older adult: Epidemiology and evolving geriatric trauma principles. Clin Geriatr Med 2013; 29:

3. Keller JM, Sciadini MF, Sinclair E, et al: Geriatric trauma: Demographics, injuries, and mortality. J Orthop Trauma 2012; 26:e161-e165

4. Barquist ES, Amortegui J, Hallal A, et al: Tracheostomy in ventilator dependent trauma patients: A prospective, randomized intention-totreat study. J Trauma 2006; 60:91-97 
5. Holevar M, Dunham JC, Brautigan R, et al: Practice management guidelines for timing of tracheostomy: The EAST Practice Management Guidelines Work Group. J Trauma 2009; 67:870-874

6. Rodriguez JL, Steinberg SM, Luchetti FA, et al: Early tracheostomy for primary airway management in the surgical critical care setting. Surgery 1990; 108:655-659

7. Arabi Y, Haddad S, Shirawi N, et al: Early tracheostomy in intensive care trauma patients improve resource utilization: A cohort study and literature review. Crit Care 2004; 8:347-352

8. Lesnik I, Rappaport W, Fulginiti J, et al: The role of early tracheostomy in blunt, multiple organ trauma. Am Surg 1992; 58:346-349

9. Rumbak MJ, Newton M, Truncale T, et al: A prospective, randomized, study comparing early percutaneous dilational tracheotomy to prolonged translaryngeal intubation (delayed tracheotomy) in critically ill medical patients. Crit Care Med 2004; 32:1689-1694

10. Maddox K, Moore E, Feliciano D: Trauma. Seventh Edition. New York, NY, The McGraw Hill Companies, 2013

11. McClave SA, Taylor BE, Martindale RG, et al; Society of Critical Care Medicine; American Society for Parenteral and Enteral Nutrition:
Guidelines for the provision and assessment of nutrition support therapy in the adult critically ill patient: Society of Critical Care Medicine (SCCM) and American Society for Parenteral and Enteral Nutrition (A.S.P.E.N.). JPEN J Parenter Enteral Nutr 2016; 40:159-211

12. Bankhead R, Boullata J, Brantley S, et al; A.S.P.E.N. Board of Directors: Enteral nutrition practice recommendations. JPEN J Parenter Enteral Nutr 2009; 33:122-167

13. U.S Department of Health and Human Services: Cost of Care. 2017. U.S. Department of Health and Human Services, Finding your path. Available at: https://longtermcare.acl.gov/costs-how-to-pay/costs-of-care.html. Accessed March 1, 2018

14. American College of Surgeons: ACS TQIP Palliative Care Best Practice Guidelines. 2017. Available at: https://www.facs.org/-/media/files/quality-programs/trauma/tqip/palliative_guidelines.ashx. Accessed March 1, 2018

15. American College of Surgeons: ACS TQIP Geriatric Trauma Management Guidelines. 2013. Available at: https://www.facs.org/-/media/files/quality-programs/trauma/tqip/geriatric_guidelines.ashx. Accessed March 1, 2018 